\title{
The clinical efficacy of Jinfeng pill in the treatment of thin endometrial infertility: a systematic review and meta-analysis
}

\author{
Xiaojuan Zhang ${ }^{1 \#}$, Yanxin Chen ${ }^{2 \#}$, Rui Zhao ${ }^{1}$, Yang Chen ${ }^{1}$, Pingting Xing ${ }^{2}$, Wen Xu ${ }^{3}$, Yu Xu ${ }^{3}$ \\ ${ }^{1}$ Department of Traditional Chinese Medicine, Hainan Women and Children's Medical Center, Haikou, China; ${ }^{2}$ Department of Obstetrics and \\ Gynecology, Haikou Hospital of Traditional Chinese Medicine, Haikou, China; ${ }^{3}$ Department of Reproductive Medicine, Hainan Modern Women \\ and Children's Hospital, Haikou, China \\ Contributions: (I) Conception and design: X Zhang; (II) Administrative support: X Zhang, Y Chen; (III) Provision of study materials or patients: R \\ Zhao, Y Chen; (IV) Collection and assembly of data: P Xing, W Xu; (V) Data analysis and interpretation: X Zhang, Y Xu; (VI) Manuscript writing: \\ All authors; (VII) Final approval of manuscript: All authors. \\ \#These authors contributed equally to this work. \\ Correspondence to: Yu Xu. Department of Reproductive Medicine, Hainan Modern Women and Children's Hospital, 18-1 Qiongzhou Avenue, Haikou \\ 571100, China. Email: xuyu34792@163.com.
}

Background To systematically evaluate the clinical efficacy of Jinfeng pill in the treatment of patients with thin endometrial infertility.

Methods: The databases of PubMed, Embase, Cochrane Library, Chinese National Knowledge Infrastructure (CNKI), Chinese Scientific Journal Database (VIP), Wanfang Science and Technology Journal Full-text Database, and Chinese Biomedical Literature Database (CBM) were used to search for articles related to Jinfeng pills in the treatment of thin endometrial infertility from database establishment to September 2021. Duplicate articles were eliminated through Endnote X9 software. Data were recorded using Stata 15.1 software, and a meta-analysis of the results was performed using fixed or random-effects models for pregnancy rate, abortion rate, endometrial thickness, and endometrial morphology type A. A funnel plot was used to evaluate the publication bias of the included literature. Article heterogeneity was judged by the $\mathrm{I}^{2}$ test, suggesting clear heterogeneity when $\mathrm{I}^{2}>50 \%$, and sensitivity analysis by excluding studies when the heterogeneity was too high.

Results: Finally, 3 articles were included. Meta-analysis showed that the pregnancy rate in the trial group was significantly higher than that in the control group; the abortion rate in the trial group was significantly lower than that in the control group; the endometrial thickening in the trial group was significantly greater than that in the control group, the above three results were statistically different. Regarding the issue of endometrial type, the results showed that there was no significant difference between the 2 groups, and the difference was not statistically significant. The funnel plot results of pregnancy rate showed that the scatter was asymmetric, suggesting that there may have been publication bias.

Discussion: Jinfeng pill can effectively improve the clinical symptoms of thin endometrial infertility, improve the pregnancy rate, reduce the abortion rate, and improve endometrial thickness, However, due to the small number of included articles and the quality of included studies, our conclusion requires verification through a larger sample and a higher quality clinical study.

Keywords: Jinfeng pill; thin endometrium; infertility; meta-analysis

Submitted Oct 21, 2021. Accepted for publication Dec 15, 2021.

doi: 10.21037/apm-21-3354

View this article at: https://dx.doi.org/10.21037/apm-21-3354 


\section{Introduction}

Thin endometrium refers to endometrial thickness below the threshold to successfully achieve pregnancy (1), and it is generally believed in clinical practice that when follicles mature, a thin endometrial thickness is less than $7 \mathrm{~mm}$ (2-4). At present, it is believed that there are four main reasons for endometrial thinning, one is that when the age is older, due to the small amount of menstruation, resulting in endometrial thinning; the second is the endometrial thinning due to hormonal factors; third, luteal function, and the endometrium is too thin; fourth, the intimal injury can cause the thinning of the endometrium. When the endometrium is too thin, the glandular epithelium grows slowly, resulting in increased arterial blood flow resistance of the uterus, which affects the receptivity of the endometrium, resulting in a decreased pregnancy rate $(5,6)$. The mechanism of thin endometrium is still not clear, and reports have indicated that it may be related to ovarian function decline, estrogen deficiency, direct endometrial damage, and long-term use of oral contraceptives (7). At present, the main clinical treatment methods for thin endometrium include estrogen drug therapy, drug therapy to improve endometrial blood flow, pelvic floor low-frequency pulse electrical stimulation therapy, traditional Chinese medicine (TCM) therapy and acupuncture treatment; however, the endometrial thickening and pregnancy outcomes have not been significantly improved (8). Thin endometrium is a common cause of infertility, and reduced endometrial thickness has an impact on endometrial tolerance. In recent years, studies have shown that Jinfeng pills have a significant effect on the treatment of thin endometrium (9-11), Jinfeng pill is currently widely used in China, but there are few relevant reports abroad, so in this meta-analysis, we hoped to include the latest studies further evaluate the efficacy of Jinfeng pills in the treatment of patients with thin endometrial infertility. We present the following article in accordance with the PRISMA reporting checklist (available at https://dx.doi.org/10.21037/apm-213354).

\section{Methods}

\section{Search strategy}

The search strategy was to search for relevant articles involving Jinfeng pills in the treatment of thin endometrial infertility from database establishment to September 2021 in 4 Chinese databases including the Chinese National
Knowledge Infrastructure (CNKI), Chinese Scientific Journal Database (VIP), Wanfang Science and Technology Journal Full-text Database, and Chinese Biomedical Literature Database (CBM) and 3 English databases of PubMed, Embase, and Cochrane Library. The search keywords were "Jinfeng pills", "infertility", "endometrial polyps", "ovulation disorders", "polycystic ovary syndrome", "pelvic inflammation and salpingitis", which were combined by a combination of free words and medical subject headings (MeSH) words.

\section{Literature screening}

Inclusion criteria: (I) definite diagnosis of thin endometrium, endometritis; (II) women aged $\leq 40$ years; (III) study type was randomized controlled trial (RCT); (IV) the clinical study of Jinfeng pill in the treatment of thin endometrial infertility is clearly mentioned in the text.

Exclusion criteria: (I) case report; (II) duplicate data publication; (III) guidelines, reviews, conference reports; (IV) other diseases; (V) abnormal changes in uterine cavity morphology caused by endometrial polyps and uterine malformations.

\section{Quality evaluation}

Quality evaluation was assessed according to the Cochrane Collaboration's risk of bias tool (12). Assessments considered the generation of random sequences, allocation concealment, blinding of participants and implementers, blinding of outcome evaluators, selective reporting, completeness of outcome data, and other biases. Literature quality evaluation was completed independently by 2 reviewers, and in the case of disagreement, a third reviewer was invited to resolve the disagreement.

\section{Data extraction}

There were 2 reviewers who independently conducted literature retrieval, and 2 reviewers independently conducted screening after retrieval. Endnote X9 software (Clarivate Analytics, Philadelphia, PA, USA) was used to process all articles in a unified manner. Then, 2 reviewers screened the studies by reading the title and abstract. After obtaining the original text, they further selected articles for inclusion in the meta-analysis according to the inclusion and exclusion criteria and extracted the required data. The extraction contents were as follows: (I) basic information 
of the literature: title, first author, region, publication time, publication journal; (II) basic characteristics of the study: total sample size, sample size in each group, course of treatment; (III) intervention indicators: different intervention methods in the trial and control group; (IV) characteristics of participants: age, duration of infertility; (V) assessment of results: pregnancy rate, abortion rate, endometrial thickness.

\section{Effect measurement}

Binary variables [pregnancy rate, miscarriage rate, endometrial morphology type A (13)] were assessed using odds ratio (OR) and their $95 \%$ confidence interval (CI); continuous variables (endometrial thickness) were assessed using standardized mean difference (SMD).

\section{Handling of data loss}

In case of any failure to provide data in the literature, the assessors first judged whether it could be obtained by calculation with the information in the text. If this was not possible, the author was contacted. If the required data was still not available, the article was excluded.

\section{Heterogeneity and sensitivity tests}

When $\mathrm{P}<0.05, \mathrm{I}^{2}>50 \%$, the results suggest heterogeneity, using a random effect model, and their sources of heterogeneity were analyzed by sensitivity analysis. When $\mathrm{P}>0.05, \mathrm{I}^{2}<50 \%$, suggesting homogeneity of the results, using a fixed-effect model. Sensitivity analysis was performed by excluding the literature one by one.

\section{Publication bias analysis}

Funnel plots were used to judge the presence of publication bias.

\section{Statistical analysis}

The software Stata 15.1 (StataCorp LLC., College Station, TX, USA) was used for analysis, and a forest plot was used to represent analysis results; the $\mathrm{I}^{2}$ and $\mathrm{Q}$ tests were used to analyze literature heterogeneity, and $\mathrm{I}^{2}>50 \%$ or $\mathrm{P}<0.05$ was used to indicate statistical difference in heterogeneity.

\section{Results}

\section{Literature search process and screening results}

A total of 72 articles were retrieved in this search, all of which were Chinese articles, without any relevant English literatures located. After duplicate checking by Endnote X9 software, 52 duplicate articles were excluded. 16 articles were excluded after reading the titles and abstracts. Then 4 articles were included. After downloading and reading the full text, a total of 3 articles were finally included in this meta-analysis. Figure 1 shows the whole process of literature screening.

\section{Basic characteristics of articles}

A total of 3 articles were included in this study, in which 268 patients participated. Table 1 shows the basic information of the included literatures.

\section{Quality assessment}

According to the Cochrane Collaboration risk of bias tool, all the included studies were low-quality studies. All articles used the random number table. All articles did not mention whether allocation concealment was performed. The blind method only mentioned the random number table. None of the articles mentioned blinding of the outcome evaluator. None mentioned whether there was an elective report. The outcome indicators were complete and did not contain other risks of bias. Figure 2 presents the risk of bias evaluation of the included studies.

\section{Combined analysis of pregnancy rate in patients with thin endometrial infertility treated with finfeng pill}

All articles (9-11) reported on the pregnancy rate in patients with thin endometrial infertility treated with Jinfeng pills. Meta-analysis showed that the pregnancy rate of the trial group was significantly higher than that of the control group ( $\mathrm{OR}=1.89,95 \% \mathrm{CI}: 1.21$ to $2.95, \mathrm{P}<0.05)$, and the difference was statistically significant (Figure 3).

Combined analysis of miscarriage rate in patients with thin endometrial infertility treated with Finfeng pill

A total of 2 articles $(9,11)$ related to abortion rate were 


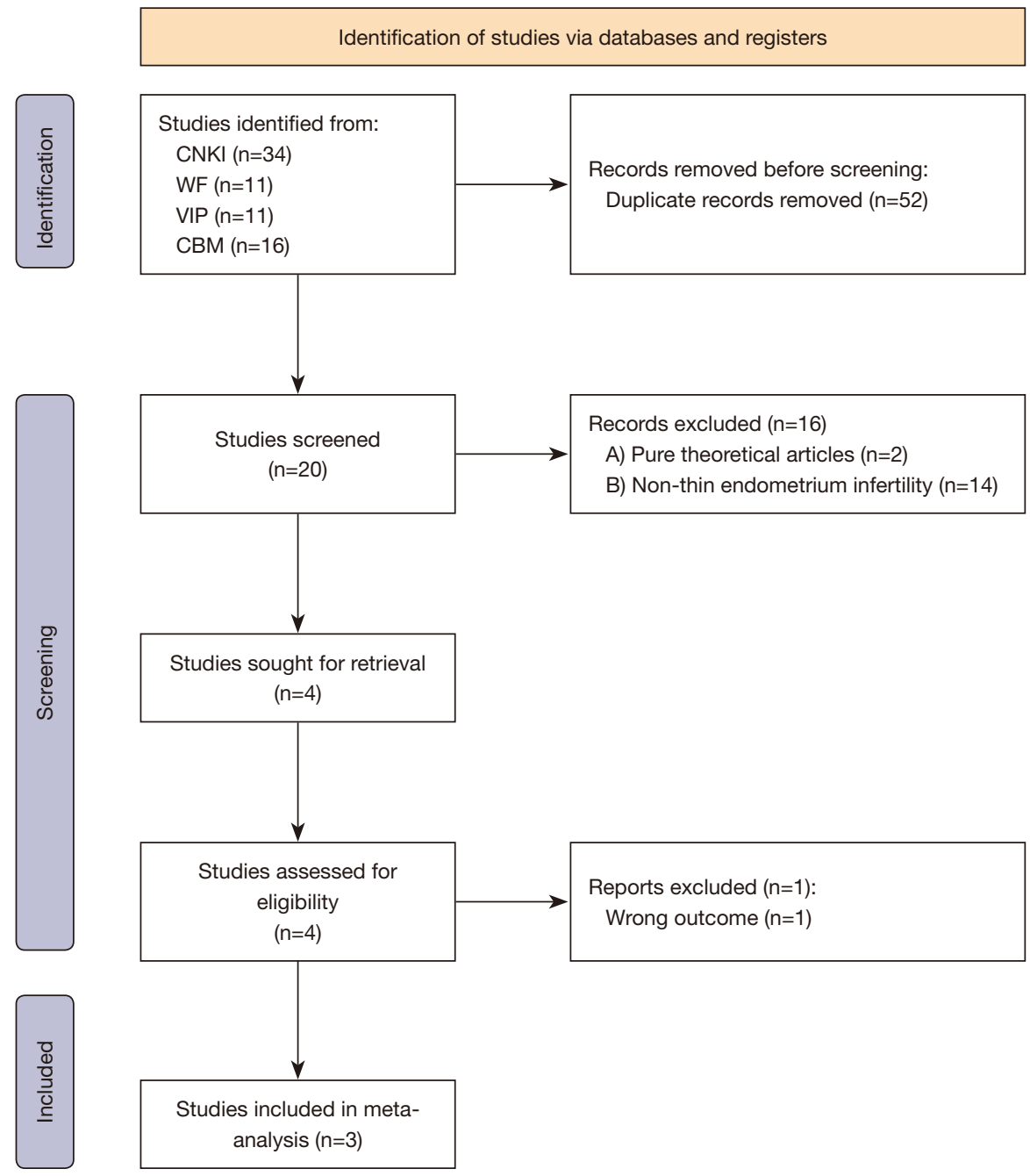

Figure 1 Literature screening flow chart.

Table 1 Basic characteristics of articles

\begin{tabular}{|c|c|c|c|c|c|c|c|}
\hline ID & \multicolumn{2}{|c|}{ Age, years } & $\begin{array}{l}\text { Sample } \\
\text { T/C }\end{array}$ & \multicolumn{2}{|c|}{ Intervention } & $\begin{array}{l}\text { Course duration } \\
\text { (menstrual cycles) }\end{array}$ & $\begin{array}{l}\text { Outcome } \\
\text { indicators }\end{array}$ \\
\hline $\begin{array}{l}\text { Zhang et al. (9), } \\
2019\end{array}$ & $33.48 \pm 7.22$ & $32.55 \pm 6.73$ & $42 / 42$ & $\begin{array}{l}\text { Estrol valtarate tablets + } \\
\text { Jinfeng pills }\end{array}$ & $\begin{array}{l}\text { Estrol valtarate } \\
\text { tablets }\end{array}$ & 3 & (1)(2)(3)(6) \\
\hline $\begin{array}{l}\text { Liu et al. (10), } \\
2019\end{array}$ & $29.3 \pm 1.8$ & $29.1 \pm 1.9$ & $52 / 52$ & $\begin{array}{l}\text { Beclomethasone + } \\
\text { Jinfeng pills }\end{array}$ & Beclomethasone & 3 & (1)(3)(4)(5) \\
\hline
\end{tabular}

T, trial group; C, control group; (1), pregnancy rate; (2), rate of abortion; (3), endometrial thickness; (4), endometrial type; (5), blood flow; (6), other. 


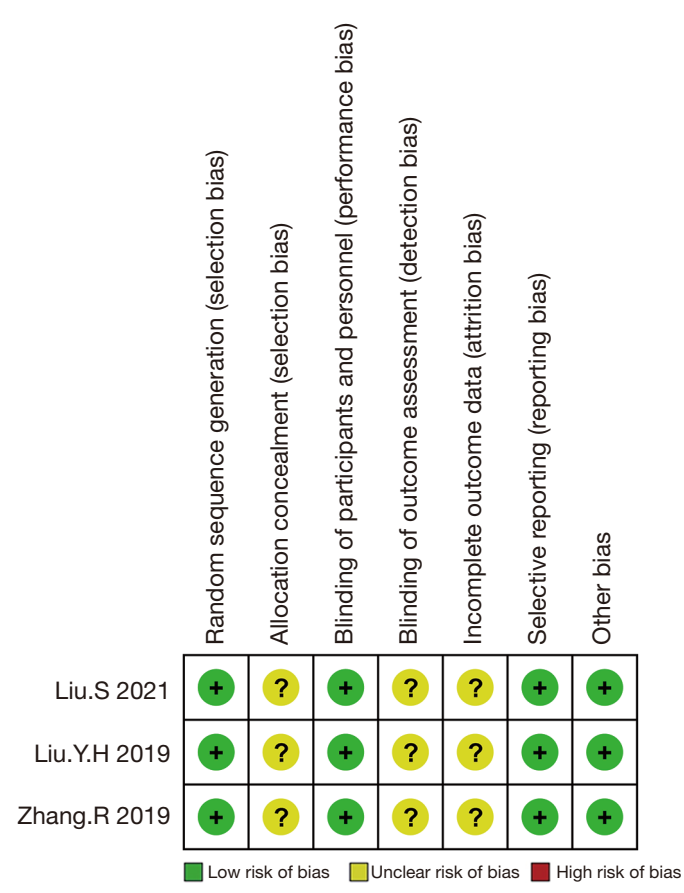

Figure 2 Risk of bias assessment diagram.

reported. Meta-analysis showed that the abortion rate in the trial group was significantly lower than that in the control group ( $\mathrm{OR}=0.23,95 \% \mathrm{CI}$ : 0.07 to $0.73, \mathrm{P}<0.05)$, and the difference had statistical significance (Figure 4).

\section{Combined analysis of endometrial thickness in patients with thin endometrial infertility treated with finfeng pill}

Endometrial thickness was mentioned in all articles (9-11). Meta-analysis showed that the endometrial thickness development in the trial group was significantly greater than that in the control group [standardized mean difference $(\mathrm{SMD})=12.59,95 \% \mathrm{CI}: 7.10$ to $18.08, \mathrm{P}<0.05]$, and the difference had statistical significance (Figure 5).

\section{Combined analysis of endometrial morphology type $A$ results in patients with thin endometrial infertility treated with Finfeng pills}

A total of 2 articles $(10,11)$ related to endometrial type were reported, and the results showed that there was no significant difference between the 2 groups $(\mathrm{OR}=1.37$, 95\% CI: 0.82 to $2.29, \mathrm{P}>0.05)$, and the difference was not statistically significant (Figure 6).

\section{Funnel plot analysis of pregnancy rate in patients with thin endometrial infertility treated with finfeng pill}

The software Stata 15.1 was used to draw a funnel plot for pregnancy rate, and the funnel plot results showed that the scatter points were asymmetric left and right, suggesting that there may be publication bias, as shown in Figure 7 .

\section{Discussion}

Thin endometrium results from damage to the basal layer of the uterus causing regenerative repair dysfunction of the endometrium (14), so that in the mid-luteal phase the endometrium is thin, which affects the receptivity of the endometrium and decrease the pregnancy rate. Thin endometrium may also cause insufficient blood supply and other problems, which can affect the normal implantation process of fertilized eggs and increase the risk of abortion. Its etiological factors are often associated with advanced age, history of uterine cavity operation, and hormonal changes (15). Clinical treatment often adjusts endometrial thickness by supplementing estrogen plus estrogen and progesterone regulation methods (16) and uses a class of drugs that can improve endometrial artery blood perfusion, to improve local uterine blood supply, promote the growth of endometrial glands, and achieve the purpose of promoting endometrial development, as well as the application of stem cells in regenerative medicine (17). Although estrogen and progestogen are the hormonal endometrial driving force, clinically, many patients in natural cycle, ovulation induction cycle, and hormone replacement cycle are unable to achieve satisfactory results in endometrial thickness, and estrogen also has great limitations in its clinical application. In the early and middle follicular phase, the application of high-dose estrogen can inhibit the production of folliclestimulating hormone, thus having malignant effects on the quality of eggs (18); at the same time, the application of high-dose estrogen may have adverse reactions such as dizziness, nausea, abnormal lipid metabolism, insomnia, breast hyperplasia, and urinary system diseases. With the prolonged application of estrogen, estrogen will also have a certain proliferative effect on the endometrium, and when the dose of estrogen is increased, the probability of small vessel or venous thrombosis stroke is heightened (19).

Jinfeng pills have the effects of supplementing qi and nourishing yin, promoting blood circulation to remove blood stasis, and supplementing qi and blood (20). The main 
Study

ID
$\%$

OR $(95 \% \mathrm{Cl}) \quad$ Weight

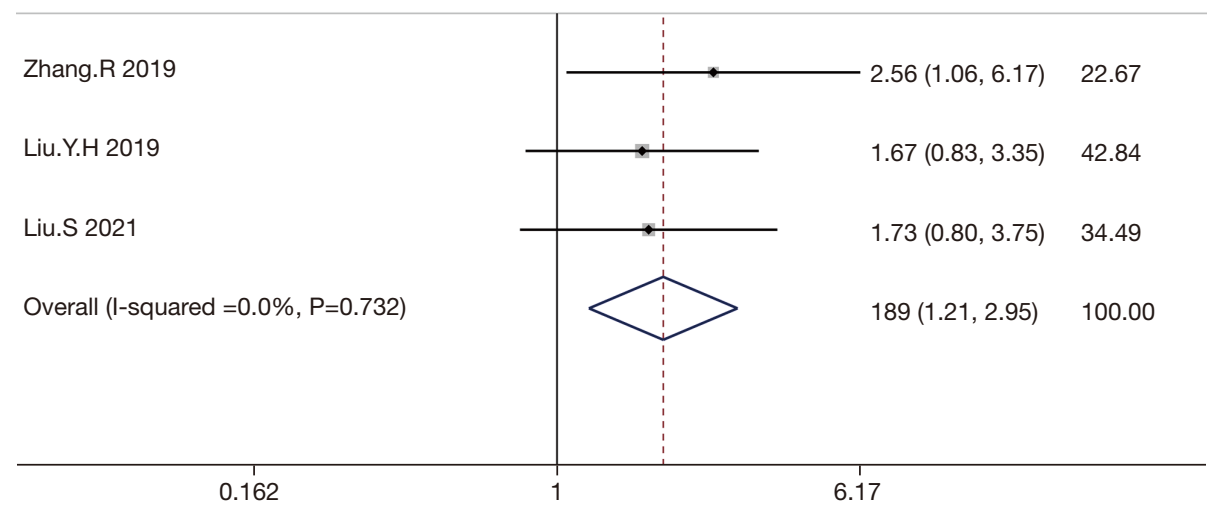

Figure 3 Forest plot for pooling of pregnancy rate results.

\begin{tabular}{|c|c|c|}
\hline Study & & $\%$ \\
\hline ID & OR (95\% Cl) & Weight \\
\hline Zhang.R 2019 & $0.27(0.07,1.05)$ & 67.59 \\
\hline Liu.S 2021 & $0.14(0.01,1.41)$ & 32.41 \\
\hline Overall (I-squared $=0.0 \%, P=0.637$ ) & $0.23(0.07,0.73)$ & 100.00 \\
\hline
\end{tabular}

Figure 4 Forest plot of combined results of abortion rate.

drug components of Jinfeng pills are concentrated waterhoneyed pills made from valuable TCM ingredients such as donkey-hide gelatin, pilose antler, Epimedium, Polygonum multiflorum Thunb., ginseng, Curculigo, Ligustrum lucidum, Leonurus heterophyllus, and Cinnamomum cassia with honey as excipients. The monarch medicine in the formula is Epimedium, the subject medicine is Polygonum multiflorum Thunb., ginseng, Ligustrum lucidum, and donkey-hide gelatin, leonurus, curculigo, and cinnamon are adjuvants, and honey is used to reconcile the various drugs while nourishing the 5 viscera. It has been shown (21) that
Curculigo, Herba Epimedii, and pilose antler can directly affect the ovary, while also influencing the ovary through the hypothalamic-pituitary gland (22). Therefore, the use of Jinfeng pills in the treatment of thin endometrial infertility can produce good efficacy, which is consistent with the analysis results of this study.

In this meta-analysis, a total of 4 articles were included, and the total sample size of the study was 268. Metaanalysis showed that the pregnancy rate in the trial group was higher than that in the control group $(\mathrm{OR}=1.89,95 \%$ CI: 1.21 to $2.95, \mathrm{P}<0.05)$, and the difference was statistically 


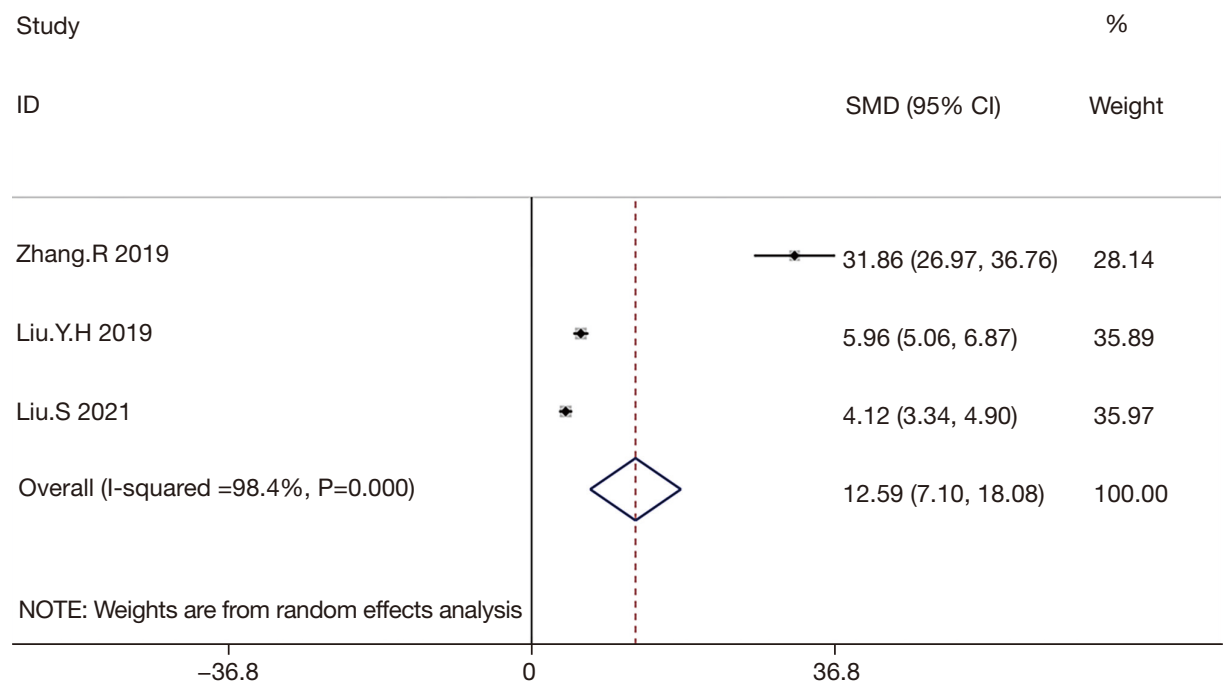

Figure 5 Forest plot of combined endometrial thickness results.

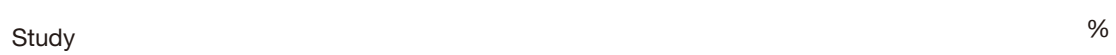

ID $\quad$ OR $(95 \% \mathrm{Cl}) \quad$ Weight

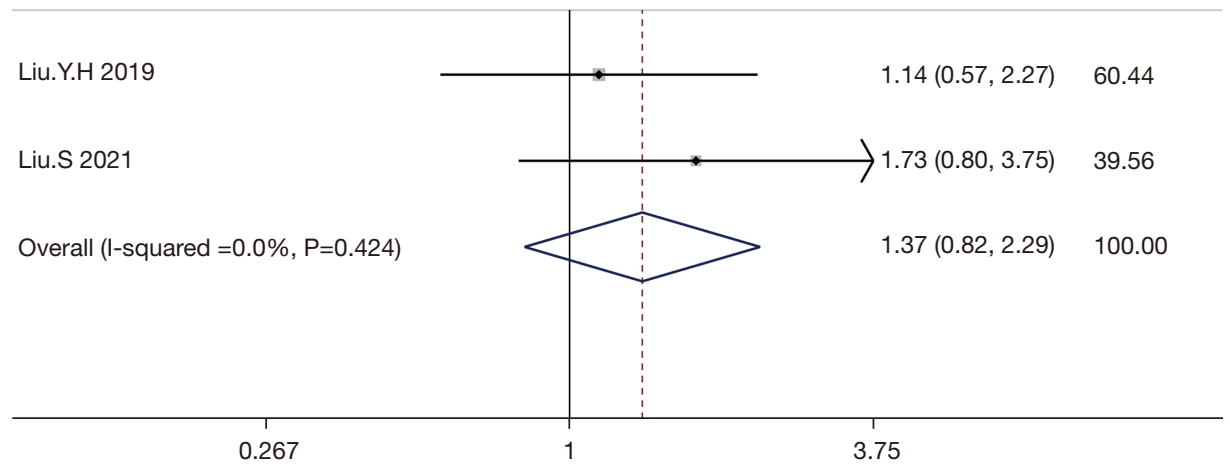

Figure 6 Forest plot of combined results of type A endometrium.

significant. The abortion rate in the trial group was lower than that in the control group ( $\mathrm{OR}=0.23,95 \% \mathrm{CI}: 0.07$ to $0.73, \mathrm{P}<0.05)$, and the difference was statistically significant. The endometrial thickness development in the trial group was greater than that in the control group $(\mathrm{SMD}=12.59$, $95 \%$ CI: 7.10 to $18.08, \mathrm{P}<0.05)$, and the difference was statistically significant. For the issue of endometrial type, the results showed that there was no significant difference between the 2 groups (OR $=1.37,95 \%$ CI: 0.82 to 2.29 , $\mathrm{P}>0.05$ ), and the difference was not statistically significant. The funnel plot results of pregnancy rate showed asymmetry around the scatter, suggesting that there may have been publication bias. According to literature included in this paper analysis, from the combined results it can be concluded that Jinfeng pill treatment of thin endometrial infertility patients has a significant effect.

This study had the following shortcomings: (I) relevant international studies on Jinfeng pills in the treatment of thin endometrial infertility were unable to be retrieved, all the included articles were of Chinese origin, and there were very few relevant Chinese reports, resulting in a small number of included articles and low sample size; (II) the 


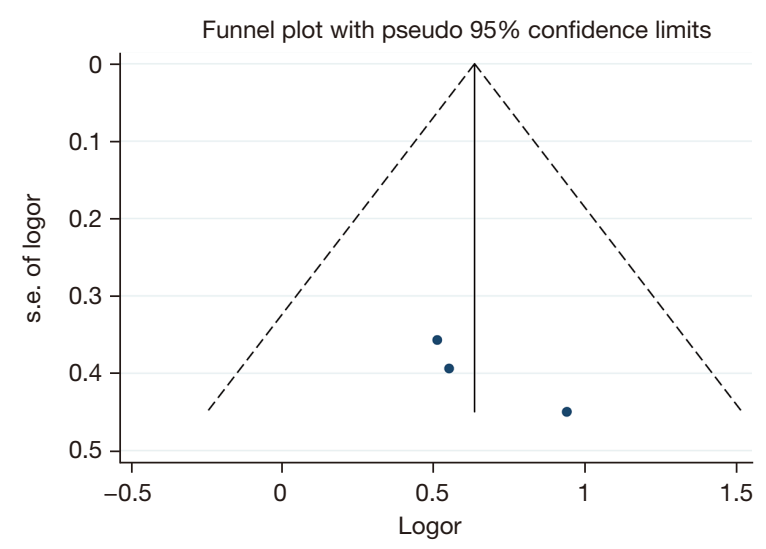

Figure 7 Funnel plot of pregnancy rate in patients with thin endometrial infertility treated with Jinfeng pills.

quality of the articles was low, meta-analysis belonged to the article reanalysis, and it was concluded that the effectiveness of the results was closely related to the quality of the included literatures, which may have caused certain bias.

\section{Conclusions}

In summary, the efficacy of Jinfeng pills in the treatment of thin endometrial infertility is worthy of validation, but due to the limitation of sample size in this study, the efficacy of Jinfeng pills in this disease still needs to be deeply studied and discussed through future efforts to include the literature of better quality and larger samples.

\section{Acknowledgments}

Funding: Project supported by Hainan Province Clinical Medical Center.

\section{Footnote}

Reporting Checklist: The authors have completed the PRISMA reporting checklist. Available at https://dx.doi. org/10.21037/apm-21-3354

Conflicts of Interest: All authors have completed the ICMJE uniform disclosure form (available at https://dx.doi. org/10.21037/apm-21-3354). The authors have no conflicts of interest to declare.

Ethical Statement: The authors are accountable for all aspects of the work in ensuring that questions related to the accuracy or integrity of any part of the work are appropriately investigated and resolved.

Open Access Statement: This is an Open Access article distributed in accordance with the Creative Commons Attribution-NonCommercial-NoDerivs 4.0 International License (CC BY-NC-ND 4.0), which permits the noncommercial replication and distribution of the article with the strict proviso that no changes or edits are made and the original work is properly cited (including links to both the formal publication through the relevant DOI and the license). See: https://creativecommons.org/licenses/by-nc-nd/4.0/.

\section{References}

1. He SM, Liang LX, Huang HF, et al. Efficacy of biometric electrical stimulation in patients with decreased ovarian reserve function. Guangdong Medicine 2013;(12):1851-5.

2. Ding H, Tian L. Progress in thin endometrium. Chinese Journal of Clinical Obstetrics and Gynecology 2017;18:568-71.

3. Riad ON, Hak AA. Assessment of endometrial receptivity using Doppler ultrasonography in infertile women undergoing intrauterine insemination. Gynecol Endocrinol 2014;30:70-3.

4. Yaman C, Mayer R. Three-dimensional ultrasound as a predictor of pregnancy in patients undergoing ART. J Turk Ger Gynecol Assoc 2012;13:128-34.

5. Miwa I, Tamura H, Takasaki A, et al. Pathophysiologic features of "thin" endometrium. Fertil Steril 2009;91:998-1004.

6. Wu JJ, Deng HT, Liu R, et al. Meta analysis of thin endometrial treatment with integrated traditional Chinese and Western medicine. Henan Traditional Chinese Medicine 2020;40:873-7.

7. Yu L, Wang SF, Ye MX, et al. New advances in thin endometrial treatment. International Journal of Reproductive Health/Family Planning 2016;35:165-9.

8. Zhao S, Shi YL. Clinical observation of Jinfeng pill combined with bioelectric stimulation for thin endometrium. Journal of Liaoning University of Traditional Chinese Medicine 2017;36:191-3.

9. Zhang R, Sun J. Clinical efficacy of Jinfeng pill combined with supplementary jiale on patients with thin endometrium infertility. The World Journal of Integrated Chinese and Western Medicine 2019;5:1263-6.

10. Liu YH, Li Y, Xiao SX, et al. Jinfeng pill combined with fendone improves pregnancy outcomes in patients 
with thin endometrial infertility. Chinese Journal of Reproductive and Contraception 2019;39:222-5.

11. Liu S, Xie KH, Wei RX, et al. Jinfeng pill combined with recombinant human growth hormone improves endometrial tolerance in patients with thin endometrial infertility. The International Journal of Reproductive Health/Family Planning 2021;40:93-7.

12. Higgins JP, Altman DG, Gøtzsche PC, et al. The Cochrane Collaboration's tool for assessing risk of bias in randomised trials. BMJ 2011;343:d5928.

13. Gonen Y, Casper RF. Prediction of implantation by the sonographic appearance of the endometrium during controlled ovarian stimulation for in vitro fertilization (IVF) J In Vitro Fert Embryo Transf 1990;7:146-52.

14. Baradwan S, Shafi D, Baradwan A, et al. The effect of endometrial thickness on pregnancy outcome in patients with Asherman's syndrome post-hysteroscopic adhesiolysis. Int J Womens Health 2018;10:77-82.

15. Wu JJ, Yan SQ, Liu R. Meta analysis of kidney and blood circulation medicine for thin endometrium. Journal of Practical Chinese Medicine 2021;32:54-9.

16. Shi HZ, Lin L, Sun LJ, et al. Effect of different estrogens on endometrial tolerance in patients with thin

Cite this article as: Zhang $\mathrm{X}$, Chen $\mathrm{Y}$, Zhao R, Chen $\mathrm{Y}$, Xing $\mathrm{P}, \mathrm{Xu} \mathrm{W}, \mathrm{Xu} \mathrm{Y}$. The clinical efficacy of Jinfeng pill in the treatment of thin endometrial infertility: a systematic review and meta-analysis. Ann Palliat Med 2021;10(12):12529-12537. doi: 10.21037/apm-21-3354 endometrium. Shanxi Medical Journal 2015;44:1050-2 .

17. Cervelló I, Gil-Sanchis C, Santamaría X, et al. Human CD133(+) bone marrow-derived stem cells promote endometrial proliferation in a murine model of Asherman syndrome. Fertil Steril 2015;104:1552-60.e1-3.

18. Liu YH, Wang X, Wang A, et al. Application of estrogen in the treatment of unknown thin endometrial type. Journal of Reproductive Medicine 2018;27:523-6.

19. Godinjak Z, Bilalovic N. Estrogen and progesterone receptors in endometrium in women with unexplained infertility. Mater Sociomed 2014;26:51-2.

20. Gao RM, Yang ZQ, Jiang JJ. 30 Clinical observations. Chinese Maternal and Child Health Research 2016;27:257-8.

21. Xu YG. Efficacy of traditional Chinese medicine plus moxibustion in 128 cases with spleen and Yang kidney deficiency polycystic ovarian syndrome. World Traditional Chinese Medicine 2014;(8):1079-82.

22. Zhang HY, Zhu FF. After Monthly 103 fewer clinical analysis. Journal of Practical Medicine 2010;26:828-30.

(English Language Editor: J. Jones) 